\title{
SEMICONTRACTIVE AND SEMIACCRETIVE NONLINEAR MAPPINGS IN BANACH SPACES
}

BY FELIX E. BROWDER

Communicated December 21, 1967

Let $X$ be a real Banach space, $U$ and $T$ mappings of a subset $G$ of $X$ into $X$. Then $U$ is said to be nonexpansive if for all $u$ and $v$ in $G$,

$$
\|U(u)-U(v)\| \leqq\|u-v\|,
$$

while $T$ is said to be accretive if for all $u$ and $v$ of $G$,

$$
(T(u)-T(v), J(u-v)) \geqq 0,
$$

where $J$ is a mapping of $X$ into its adjoint space $X^{*}$ such that for all $u$ in $X,(J(u), u)=\|u\|^{2}$ and $\|J(u)\|=\|u\|$.

In some recent papers ([7], [8], [9]), we have presented an existence theory for solutions of nonlinear functional equations in uniformly convex Banach spaces $X$ involving nonexpansive and accretive mappings. These results were obtained by interweaving the fixed point theory of nonexpansive mappings with the theory of initial value problems for differential equations in $X$ involving accretive operators. It is our object here to sharpen this theory and to use the sharpened form to extend the preceding results to more general classes of operators obtained by compact perturbation from nonexpansive or accretive operators. When $X$ is a Hilbert space (or, more generally, has a weakly continuous duality mapping), such results were obtained earlier by the writer in [1], [2], [4]. The methods used there involving monotone operators do not apply in our more general context.

We begin by defining two basic classes of nonlinear mappings, the first generalizing the mappings of the form $U+C$ with $U$ nonexpansive and $C$ completely continuous, and the second, the mappings of the form $T+C$ with $T$ accretive and $C$ completely continuous. (We recall that a map $C$ of $X$ into $X$ is said to be completely continuous if it carries weakly convergent sequences in $X$ into strongly convergent sequences in $X$.)

Definition 1. Let $X$ be a Banach space, $G$ a subset of $X, U$ a mapping of $G$ into $X$. Then $U$ is said to be semicontractive if there exists a mapping $V$ of $G \times G$ into $X$ such that $U(u)=V(u, u)$ for $u$ in $G$, while:

(a) For each fixed $v$ in $G, V(\cdot, v)$ is nonexpansive from $G$ to $X$.

(b) For each fixed $u$ in $G, V(u, \cdot)$ is completely continuous from $G$ 
to $X$, uniformly for $u$ in bounded subsets of $G$, (i.e. if $v_{j}$ converges weakly to $v$ in $G$ and $\left\{u_{j}\right\}$ is a bounded sequence in $G$, then

$$
\left.V\left(u_{j}, v_{j}\right)-V\left(u_{j}, v\right) \rightarrow 0 \text {, strongly in } G\right) \text {. }
$$

Definition 2. Let $X$ be a Banach space, $G$ a subset of $X, T$ a map of $G$ into $X$. Then $T$ is said to be semiaccretive if there exists a mapping $S$ of $G \times X$ into $X$ such that $T(u)=S(u, u)$ for $u$ in $G$, while:

(a) For each fixed $v$ in $X, S(\cdot, v)=S_{v}$ is an accretive map from $G$ to $X$ such that either $G=X$ and $S_{v}$ is continuous or $S_{v}=S_{1, v}+S_{0, v}$ where $S_{1, v}+I$ has all of $X$ as its range and $S_{0, v}$ is the restriction to $G$ of a bounded continuous accretive mapping of $X$ into $X$.

(b) For each fixed $u$ in $G, S(u, \cdot)$ is completely continuous from $X$ to $X$, uniformly for $u$ in bounded subsets of $G$.

Our basic results are the following theorems:

Theorem 1. Let $X$ be a uniformly convex Banach space, $G$ a closed bounded convex subset of $X$ with 0 in the interior of $G$. Let $U$ be a semicontractive mapping of $G$ into $X$ such that for each $x$ in the boundary of $G, U(x) \neq \lambda x$ for any $\lambda>1$. Then $U$ has a fixed point in $G$.

Theorem 2. Let $T$ be a semiaccretive mapping of $G$ into $X$ as defined in Definition 2, where $X$ and $X^{*}$ are uniformly convex. Suppose that $T^{-1}(B)$ is bounded for each bounded set $B$. Then the range of $T$ is all of $X$.

Theorems 1 and 2, as well as other more precise results, follow from the following basic properties of semicontractive and semiaccretive operators given in Theorems 3 and 4 , together with the more general existence theorems, Theorems 5 and 6.

Theorem 3. Let $X$ be uniformly convex, $G$ a closed bounded convex subset of $X, U$ a semicontractive mapping of $G$ into $X$. Then:

(a) $(I-U)$ is demiclosed, i.e. if $u_{j}$ converges weakly to $u_{0}$ in $G$ and $(I-U) u_{j}$ converges strongly to $w$, then $(I-U)\left(u_{0}\right)=w$.

(b) $(I-U)(G)$ is closed in $X$.

TheOREM 4. Let $X$ be uniformly convex with $X^{*}$ uniformly convex, $T$ a semiaccretive mapping from a subset $G_{0}$ of $X$ into $X$ (as in Definition 2). Then $T$ is demiclosed and for any closed bounded convex subset $G$ of $X, T\left(G \cap G_{0}\right)$ is closed in $X$.

TheOREM 5. Let $X$ be a Banach space, $G$ a closed bounded convex subset of $X$ having 0 in its interior, $U$ a mapping of $G$ into $X$ such that for each $x$ on the boundary of $G, U(x) \neq \lambda x$ for any $\lambda>1$. Suppose that 
for a given constant $k \leqq 1$ and a mapping $V$ of $G \times G$ into $X, U(u)$ $=V(u, u)$ for all $u$ in $G$ while

$$
\|V(u, w)-V(v, w)\| \leqq k\|u-v\|,(u, v, w \in G),
$$

and the map $u \rightarrow V(\cdot, u)$ is compact from $G$ to the space of maps from $G$ to $X$ with the uniform metric.

Then:

(a) If $k<1, U$ has a fixed point in $G$.

(b) If $k \leqq 1$ and $(I-U)(G)$ is closed in $X$, then $U$ has a fixed point in $G$.

Theorem 6. Let $X$ be a Banach space with $X^{*}$ uniformly convex, $T$ a semiaccretive mapping from a subset of $X$ into $X$ (in the sense of Definition 2). Suppose that $T^{-1}(B)$ is bounded for each bounded ball $B$ in $X$, and that for each ball about the origin, $T(B)$ is closed in $X$.

Then the range of $T$ is the whole space $X$.

Theorem 1 follows from the Theorems 3 and 5, while Theorem 2 follows from Theorems 4 and 6.

Proof of Theorem 3. For any demiclosed map $T$ and any weakly compact subset $G, T(G)$ is closed. Hence it suffices to prove $(I-U)$ demiclosed. If we replace $U$ by $U+w_{0}, w_{0}$ fixed, we may take $w=0$, and we have $u_{j} \rightarrow u_{0}$ weakly, $(I-U) u_{j} \rightarrow 0$ strongly, and it suffices to show that $(I-U)\left(u_{0}\right)=0$. By condition (b) of Definition $1, V\left(u_{j}, u_{j}\right)$ $-V\left(u_{j}, u\right) \rightarrow 0$ strongly. Hence $u_{j}-V\left(u_{j}, u\right) \rightarrow 0$ strongly. Replacing $U$ by $V(\cdot, u)$, we may assume without loss of generality that $U$ is nonexpansive.

Since $X$ is uniformly convex, for each $\epsilon>0$, there exists a largest $\xi(\epsilon)>0, \xi(\epsilon) \leqq \epsilon$ such that if for any three elements $x, u$, and $v$ of $X$ and any $\lambda$ with $0 \leqq \lambda \leqq 1$, we have $\|x-u\| \leqq\left\|u_{\lambda}-u\right\|+\xi(\epsilon),\|x-v\|$ $\leqq\left\|u_{\lambda}-v\right\|+\xi(\epsilon)$, with $u_{\lambda}=\lambda u+(1-\lambda) v$, then

$$
\left\|x-u_{\lambda}\right\| \leqq \epsilon\|u-v\| \text {. }
$$

We assume diam $G \leqq 1$ and following a device of Göhde [11], for a given $\epsilon_{0}>0$, we let $\left\{\epsilon_{j}\right\}$ be the sequence defined recursively by $\epsilon_{j}=\xi\left(\epsilon_{j-1}\right)$. We note that if $\|u-U(u)\| \leqq \epsilon_{j},\|v-U(v)\| \leqq \epsilon_{j}$, and let $u_{\lambda}=\lambda u+(1-\lambda) v$ for $\lambda$ in $[0,1]$, then $\left\|U\left(u_{\lambda}\right)-u\right\| \leqq\left\|U\left(u_{\lambda}\right)-U(u)\right\|$ $+\|u-U(u)\| \leqq\left\|u_{\lambda}-u\right\|+\epsilon_{j} . \quad$ Similarly, $\quad\left\|U\left(u_{\lambda}\right)-v\right\| \leqq\left\|u_{\lambda}-v\right\|+\epsilon_{j}$. Since $\epsilon_{j}=\xi\left(\epsilon_{j-1}\right)$, it follows that $\left\|u_{\lambda}-U\left(u_{\lambda}\right)\right\| \leqq \epsilon_{j-1}$.

By thinning out the sequence $\left\{u_{j}\right\}$, we may assume that for each $j,\left\|u_{j}-U\left(u_{j}\right)\right\| \leqq \epsilon_{j}$. If $y$ is any element of the convex span of the sequence $\left\{u_{j}\right\}$, after this thinning out process has been carried through, we assert that $\|y-U(y)\| \leqq \epsilon_{0}$. Indeed, for $y$ in the convex span of 
$\left[u_{j}, \cdots, u_{k}\right]$ (written with ascending indices), $\|y-U(y)\| \leqq \epsilon_{j-1}$. For two elements $\left[u_{j}, u_{k}\right]$, this follows from the preceding paragraph. If it is true for the $s$ elements $\left[u_{j_{1}}, \cdots, u_{k}\right]$, it is true for $(s+1)$ elements $\left[u_{j}, u_{j_{1}}, \cdots, u_{k}\right]$ since an element of the convex span of the latter set is a convex linear combination of $u_{j}$ and of an element of the convex span of $\left[u_{j_{1}}, \cdots, u_{k}\right]$. Since $u_{0}$ is the weak limit of the thinned-out sequence $\left\{u_{j}\right\}$, it lies in the convex span of the sequence. Hence $\left\|u_{0}-U\left(u_{0}\right)\right\| \leqq \epsilon_{0}$. Since $\epsilon_{0}>0$ was arbitrary, it follows that $u_{0}=U\left(u_{0}\right)$.

Q.E.D.

Proof of Theorem 4. As in the proof of Theorem 3, it suffices to prove that $T$ is demiclosed, and we may replace $T$ by $S\left(\cdot, u_{0}\right)$ and assume that $T$ is accretive and satisfies condition (a) of Definition 2. By the results of [7], for each $v$ in $G_{0}$, the domain of $T$, the initial value problem

$$
d u / d t=-T(u), \quad t \geqq 0 ; u(0)=v,
$$

has one and only one weakly continuously differentiable solution $u$ on $R^{+}$, the mapping $U(t)$ for each $t \geqq 0$ given by $U(t) v=u(t)$ is a nonexpansive map of $G_{0}$ into $X$, and for all $t \geqq 0,\|T(u(t))\| \leqq\|T(v)\|$.

Suppose then that $u_{j} \rightarrow u_{0}$ weakly and that $T\left(u_{j}\right) \rightarrow 0$ strongly in $X$. If $G$ is the convex closure of the sequence $\left\{u_{j}\right\}$, it suffices to show that there exists an element $u^{\prime}$ in the closed bounded convex subset $G$ for which $T\left(u^{\prime}\right)$ is defined and equals 0 . As we have already noted in [8], such an element $u^{\prime}$ is precisely one lying in the common fixed point set of the mappings $U(t), t>0$. To show that this common fixed point set is nonvoid, it suffices to show, since the mappings $U(t)$ commute, that each $U(t)$ has a fixed point in $G$. However,

$$
\left\|u_{j}-U(t) u_{j}\right\| \leqq \int_{0}^{t}\left\|T(U(t)) u_{j}\right\| d t \leqq t\left\|T\left(u_{j}\right)\right\| \rightarrow 0 \quad(j \rightarrow+\infty) .
$$

Applying Theorem 3, we see that $U(t)$ has a fixed point in G. Q.E.D.

Proof of Theorem 5. We note first that conclusion (b) of Theorem 5 follows from conclusion (a). Indeed, if $U$ is a mapping satisfying the conditions of (b), then for each $k$ with $0<k<1$, the mapping $U_{k}(x)=k U(x)$ satisfies the condition of (a) with $V_{k}(u, v)=k V(u, v)$, and on the boundary of $G, U_{k}(x)=\lambda x$ implies that $U(x)=\lambda k^{-1} x$. Applying the conclusion of (a), we see that $U_{k}$ has a fixed point $u_{k}$ in $G$. As $k \rightarrow 1, u_{k}-U\left(u_{k}\right)=(k-1) U\left(u_{k}\right) \rightarrow 0$. If $(I-U)(G)$ is closed, it follows that $U$ has a fixed point in $G$.

The proof of conclusion (a) follows a variant of the argument of [5]. For each $t$ with $0 \leqq t \leqq 1$, we set $U_{t}(x)=t U(x)$. Our hypothesis on the nonexistence of eigenfunctions of $U$ on the boundary of $G$ with 
eigenvalues $\lambda>1$, implies that either $U$ has a fixed point on the boundary of $G$, (the trivial case), or none of the mappings $U_{t}$ have fixed points on the boundary of $G$. For each $t$ and each $w$ in $G$, the mapping $V_{t}(\cdot, w)$ is a strict contraction on $G$ with values in $X$. Hence the image of the interior of $G$ under the mapping $I-V_{t}(\cdot, w)$ is an open subset $G_{t, w}$ of $X$ and $\left(I-V_{t}(\cdot, w)\right)$ maps the boundary of $G$ on the boundary of $G_{t, w}$. For each $v$ in $\bar{G}_{t, w}$, there exists exactly one element $u$ of $G$ such that $u-V_{t}(u, w)=v$. We denote this element by $C_{t, v}(w)$. Since the map $w \rightarrow V(\cdot, w)$ is a compact map of $G$ into the space of maps from $G$ to $X$ with the uniform metric, $C_{t, v}$ is a compact map of $G$ into $X$ for each fixed $t$ and $v$, and indeed the union of the images over all $t$ in $[0,1]$ and all $v$ in $G$ of the maps $C_{t, v}$ is relatively compact in $X$.

For each $t$ in $[0,1]$, we now let $\psi_{t}=\left\{w \mid w \in G, 0 \in \bar{G}_{t, w}\right\}$ and for each $w$ in $\psi_{t}$, we define $Q_{t}(w)=C_{t, 0}(w)$. Similarly, we define $\psi$ as the subset of $X \times I$ consisting of those points $[t, w]$ such that $w$ lies in $\psi_{t}$, and we set $Q(t, w)=Q_{t}(w)$. We note that if $w$ lies in the boundary of $\psi_{t}$, then $C_{t, 0}(w)$ is well defined and lies on the boundary of $G$. Hence $Q$ may be extended by continuity to a compact mapping of the closure of $\psi$ into $X$, and maps all points of the boundary of $\psi$ into the boundary of $G$. Moreover, $\psi$ is an open subset of $X \times I$, since if 0 lies in the image of $I-V_{t}(\cdot, w)$ acting on the interior of $G$ for a given pair $[t, w]$, then for $[s, z]$ near $[t, w]$, the equation $u-V_{s}(u, z)=0$ must have a solution $u$ in the interior of $G$ since the image of the interior of $G$ by the homeomorphism $I-V_{s}(\cdot, z)$ must contain an entire component of $X-\left(I-V_{s}(\cdot, z)\right)$ (bdry $\left.G\right)$. By choosing $[s, z]$ near $[t, w]$, we can ensure that the boundary image above does not intersect a given ball about 0 while the image of $C_{t, 0}(w)$ under $\left(I-V_{s}(\cdot, z)\right)$ lies in that ball. Hence the whole ball including the point 0 is covered by the image of $\left(I-V_{s}(\cdot, z)\right)$.

To complete the proof, we apply the Leray-Schauder theory [14]. For $t=0, Q_{0}$ is the constant map 0 defined on $\psi_{0}=G$, and the degree of $\left(I-Q_{0}\right)$ on $\psi_{0}$ must equal +1 . For all $t$ and all $x$ on the boundary of $\psi_{t}, Q_{t} x$ is never equal to $x$, since if it were, we should have $x-V_{t}(x, x)=x-U_{t}(x)=0$ with $x=Q_{t} x$ lying on the boundary of $G$. Hence $Q_{1}$ has a fixed point in $\psi_{1}$, which implies that $x-U(x)=0$ has a solution in $G$.

Q.E.D.

The proof of Theorem 6, which is another variant of the argument of [5], will be given in detail elsewhere.

In conclusion, we note that the result of Theorem 3 yields the following generalization of the result of Browder-Petryshyn [10]: 
TheOREM 7. Let $X$ be a uniformly convex Banach space, $G$ a closed bounded convex subset of $X, U$ a nonexpansive mapping of $G$ into $G$. For a constant $t$ with $0<t<1$, let $U_{t}=t U+(1-t) I$. Suppose that $U$ has at most one fixed point $y$ in $G$. Then for each $x_{0}$ in $G$, the sequence $\left(U_{t}\right)^{n} x_{0}$ converges weakly to $y$ in $X$.

Proof of Theorem 7. Let $x_{n}=\left(U_{t}\right)^{n} x_{0}$ for a given $x_{0}$ and $n \geqq 1$. To show that $x_{n}$ converges weakly to $y$, it suffices to show that each weakly convergent subsequence of $\left\{x_{n}\right\}$ converges to a fixed point of $U$. It suffices by the demiclosedness of $U$ to show that $(I-U)\left(x_{n}\right) \rightarrow 0$. Since $U_{t}$ is also nonexpansive and $y$ is a fixed point of $U_{t},\left\|x_{n}-y\right\|$ is nonincreasing in $n$, and hence converges. On the other hand, $x_{n+1}-y$ $=t\left(U\left(x_{n}\right)-U(y)\right)+(1-t)\left(x_{n}-y\right)$, where $\left\|U\left(x_{n}\right)-U(y)\right\| \leqq\left\|x_{n}-y\right\|$. By the uniform convexity of $X$, it follows that $U\left(x_{n}\right)-x_{n} \rightarrow 0$. Q.E.D.

\section{BIBLIOGRAPHY}

1. F. E. Browder, Existence of periodic solutions for nonlinear equations of evolution, Proc. Nat. Acad. Sci. U.S.A. 53 (1965), 1100-1103.

2. - Fixed point theorems for noncompact mappings in Hilbert spaces, Proc. Nat. Acad. Sci. U.S.A. 53 (1965), 1272-1276.

3. - Nonexpansive nonlinear operators in a Banach space, Proc. Nat. Acad. Sci. U.S.A. 54 (1965), 1041-1044.

4. - Fixed point theorems for nonlinear semicontractive mappings in Banach spaces, Arch. Rational Mech. Anal. 21 (1966), 259-269.

5. - Nonlinear operators in Banach spaces, Math. Ann. 162 (1966), 280-283. 476.

6. - - Nonlinear accretive operators, Bull. Amer. Math. Soc. 73 (1967), 470-

7. - Nonlinear operators and nonlinear equations of evolution in Banach spaces, Proc. Sympos. on Nonlinear Func. Anal., April 1968. (to appear).

8. - Nonlinear equations of evolution and nonlinear accretive operators in Banach spaces, Bull. Amer. Math. Soc. 73 (1967), 867-874.

9. - Nonlinear mappings of nonexpansive and accretive type in Banach spaces, Bull. Amer. Math. Soc. 73 (1967), 875-882.

10. F. E. Browder and W. V. Petryshyn, The solution by iteration of nonlinear functional equations in Banach spaces, Bull. Amer. Math. Soc. 72 (1966), 571-575.

11. D. Göhde, Zum Prinzip der kontraktiven Abbildung, Math. Nachr. 30 (1966), 251-258.

12. T. Kato, Nonlinear semigroups and evolution equations, (to appear).

13. W. A. Kirk, $A$ fixed point theorem for mappings which do not increase distance, Amer. Math. Monthly 72 (1965), 1004-1006.

14. J. Leray and J. Schauder, Topologie et equations fonctionelles, Ann. Sci. Ecole Norm. Sup. Paris 51 (1934), 45-78.

15. Z. Opial, Weak convergence of the sequence of successive approximants for nonexpansive mappings in Banach spaces, Bull. Amer. Math. Soc. 73 (1967), 591-597.

University of Chicago 